\title{
Phosphine in soils, sludges, biogases and atmospheric implications-a review
}

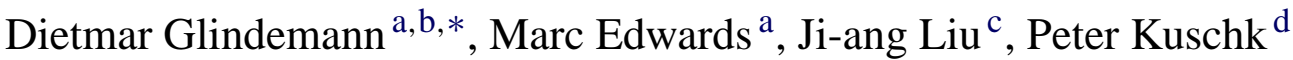 \\ ${ }^{a}$ Virginia Tech, 415 Durham Hall, Blacksburg, VA 24061, USA \\ b Institute for Animal Hygiene and Veterinary Public Affairs, University of Leipzig, \\ Semmelweisstrasse 4, D-04103 Leipzig, Germany \\ ${ }^{\mathrm{c}}$ Chinese Acad. Sci., State Key Lab. Environm. Aquat. Chem. (SKLEAC), Beijing 100085, PR China \\ ${ }^{\mathrm{d}}$ Centre for Environmental Research Leipzig-Halle Ltd., Permoser Str. 15, D-04318 Leipzig, Germany
}

Accepted 10 January 2005

\begin{abstract}
This is a review of previously published and unpublished results of research into the occurrence of phosphine $\left(\mathrm{PH}_{3}\right)$ in the environment in the form of matrix bound phosphine in soils, aquatic sediments and sludges (range $\mathrm{n} \mathrm{kg}^{-1}$ to $\mu \mathrm{g} \mathrm{kg}^{-1}$ ), free phosphine in formed biogases (range $\mathrm{ng} \mathrm{m}^{-3}$ to $\mathrm{\mu g} \mathrm{m}^{-3}$ ) and in the atmosphere (range $\mathrm{pg} \mathrm{m}^{-3}$ to $\mathrm{ng} \mathrm{m}^{-3}$ ).

The reviewed data support the hypothesis of the existence of a small gaseous link in the phosphorus cycle, which could become important over the long term.

Matrix-bound phosphine in soils can be interpreted as a stationary state concentration of phosphine between production and consumption. This phosphine turnover within the soil may be important even if the stationary state concentration (matrix-bound phosphine) is small. Under such circumstances, a slow migration process of phosphine in the interstitial gas sphere of soils is possible. Such a process would influence the balance of phosphorus in agricultural and wetland soil.

The detection of easily oxidizable phosphine as a ubiquitous trace gas in the atmosphere can be interpreted as the residue of an important turnover of phosphine between widely distributed emission sources and sinks such as soils and sediments. The atmosphere can carry gaseous phosphorus to remote places.
\end{abstract}

(C) 2005 Elsevier B.V. All rights reserved.

Keywords: Phosphine; Phosphorus cycling; Soil; Sediment; River; Lake; Manure; Biogas; Atmosphere

\footnotetext{
* Corresponding author. Tel.: +1 5402312146 ; fax: +1 5402317916 .

E-mail address: dglinde@al.com (D. Glindemann).
}

\section{Introduction}

Phosphine $\left(\mathrm{PH}_{3}\right)$, a volatile and toxic molecule containing phosphorus in the oxidation state 3 , is produced by humans for industrial application and as a pesticide. It is also contained in several waste media (WHO, 1988). 
Regarding its natural occurrence, phosphine is only common in the methane and hydrogen atmosphere of the gas planets (Prinn and Lewis, 1975). Phosphine is rare in the earth's environment because it is easily oxidized by air (WHO, 1988), and is difficult to produce. New analytical developments made it possible to detect small amounts of phosphine in soil, sludge and biogases in the environment (Devai et al., 1984, 1988; Devai and DeLaune, 1995; Eismann et al., 1997; Gassmann and Glindemann, 1993; Gassmann and Schorn, 1993; Gassmann, 1994; Glindemann and Bergmann, 1995; Glindemann et al., 1996a; Han et al., 2000; Iverson, 1968; Liu et al., 1999). Tsubota (1959) found not phosphine but phosphites as reduced phosphorus compounds in a Japanese paddy field. Phosphine was even found to be a worldwide trace component in the earth's atmosphere (Gassmann and Glindemann, 1996; Glindemann et al., 1996a).

Several independent reports claim that phosphine can be produced by biochemical processes in the laboratory (Cao et al., 2000; Devai et al., 1984, 1988; Eismann et al., 1997; Gassmann and Glindemann, 1993; Iverson, 1968; Jenkins et al., 2000). Most of these results have been summarized in a review (Roels and Verstraete, 2001). Therefore, biochemical processes (such as the bacterial reduction of phosphates or natural organophosphorus compounds) would support a gaseous link to the phosphorus cycle in the environment.

This is contrary to the common assumption that in contrast to carbon, nitrogen, sulphur, oxygen, and some other elements, phosphorus can only cycle in different non-volatile forms of phosphates and phosphonates (Graham and Duce, 1979; Bolin and Cook, 1983). It is unquestionable that the finding of phosphine is a scientific curiosity. Phosphine is also connected with the ignition of swamp lights (Ignis Fatuus), spontaneous human combustion (SHC), the phosphorescence of graves and fire-breathing dragons (Emsley, 2000).

An important question is whether phosphine gas is an important component of the geochemical cycling of phosphorus in the environment. One of the more practical questions is whether phosphine is a significant pool of phosphorus nutrient in agricultural farmland, were total $\mathrm{P}$ can be very high (eutrophication), or in highland wetlands, were $\mathrm{P}$ can be limiting for the biosphere.

We present here a review of published and previously unpublished numbers on the occurrence of phosphine in soils (the source or sink), in developed gases (the possible emission medium) and in the atmosphere (the carrier of phosphine).

\section{Definitions and generalized reviewed methods}

Free phosphine is the concentration of phosphine, which is spontaneously free measurable in gas samples such as biogas or air. Its unit is mass phosphine-phosphorus per $\mathrm{m}^{3}$, and it is measured using gas chromatography.

Matrix-bound phosphine is the concentration of phosphine in soils, sediments, sludges and other condensed media. Its unit is mass phosphine-phosphorus per $\mathrm{kg}$ soil, etc., and it is measured similarly to the analysis of phosphine residue in grain (Nowicki, 1978) fumigated with phosphides or phosphine (anaerobic digestion of the soil with a strong mineral acid $\left(\mathrm{H}_{2} \mathrm{SO}_{4}\right.$, $\mathrm{HCl})$ or alkaline solution $(\mathrm{NaOH})$ and subsequent quantification of phosphine in the reaction gas). More details of the methods can be found in the references. This chemical digestion can hydrolyze non-volatile phosphides into phosphine gas. It can also, however, liberate phosphine from a form in which it is adsorbed in the matrix (therefore the name matrix phosphine) into phosphine gas. Therefore, matrix-bound phosphine is a function of the applied digestion method, and does not necessarily refer to the pre-existence of phosphine gas in the matrix, because it could be in the form of solid phosphides. Phosphides, on the other hand, can hydrolyze in biological aquatic media (Glindemann et al., 1998) to form free phosphine gas.

\section{Reviewed results and discussion}

In soils, the concentration of phosphine is in the range $\mathrm{ng} \mathrm{kg}^{-1}$ to $\mu \mathrm{g} \mathrm{kg}^{-1}$ (Table 1). This is very low compared with their phosphate content, which is generally much higher.

Eismann et al. (1997) simulated phosphine production and consumption by soil samples in the laboratory, showing that biological metabolites such as sulfide, glucose, formate, and pyrogallol can accelerate the release of phosphine from the soil. Generally, however, the consumption of phosphine by soil is faster than 
Table 1

Matrix-bound phosphine in aquatic sediments, soils, stone minerals, and animal/human waste which is placed on soils as fertilizer

\begin{tabular}{|c|c|c|c|c|c|c|}
\hline \multirow[t]{2}{*}{ Sample matrix } & \multirow[t]{2}{*}{ Location } & \multirow{2}{*}{$\begin{array}{l}\text { Independent } \\
\text { samples }(n)\end{array}$} & \multicolumn{2}{|c|}{ Phosphine $\left(\mathrm{ng} \mathrm{kg}^{-1}\right)$} & \multirow{2}{*}{$\begin{array}{l}\text { Digesting } \\
\text { agent }\end{array}$} & \multirow[t]{2}{*}{ References } \\
\hline & & & Average & Range & & \\
\hline Water, bottom, fresh & Hamburg harbour & 10 locations & 60 & $0-387$ & & \\
\hline Sediment, surface & Hamburg harbour & 10 locations & 99 & $43-201$ & & \\
\hline Sediment, subsurface & Hamburg harbour & 10 locations & 216 & $93-753$ & $10 \% \mathrm{NaOH}$ & Gassmann (1994) \\
\hline Water, bottom, salty & German bight & 18 locations & 8 & $0-28$ & & \\
\hline Sediment, surface & German bight & 18 locations & 770 & $158-1923$ & & \\
\hline Sediment, subsurface & German bight & 18 locations & 853 & $81-2217$ & & \\
\hline Sediment, surface & Hamburg harbour & 10 locations & 24 & $0.2-56.1$ & $10 \% \mathrm{NaOH}$ & $\begin{array}{l}\text { Gassmann and Schorn } \\
\text { (1993) }\end{array}$ \\
\hline Soil, industrial area & Germany & 2 locations & 60845 & $18440-103250$ & $1 \mathrm{~N} \mathrm{H}_{2} \mathrm{SO}_{4}$ & Eismann et al. (1997) \\
\hline Soil, rural area & Germany & 5 locations & 4476 & 840-17050 & & \\
\hline Sediment, fresh & $\begin{array}{l}\text { Elster river, } \\
\text { Germany }\end{array}$ & $\begin{array}{l}26 \text { (4 locations, } \\
5 \text { seasons) }\end{array}$ & 227 & $4-1140$ & & $\begin{array}{l}\text { Glindemann (not } \\
\text { published) }\end{array}$ \\
\hline $\begin{array}{l}\text { Sediment, landfilled } \\
\text { over } 1 \text { year }\end{array}$ & $\begin{array}{l}\text { Elster river, } \\
\text { Germany }\end{array}$ & $\begin{array}{l}5 \text { (1 location, } 5 \\
\text { depths) }\end{array}$ & 137 & $110-165$ & & \\
\hline $\begin{array}{l}\text { Sediment, landfilled, } \\
\text { after } 4 \text { weeks of full } \\
\text { scale acidic } \\
\text { leaching }\end{array}$ & $\begin{array}{l}\text { Elster river, } \\
\text { Germany }\end{array}$ & $\begin{array}{l}5 \text { (1 locations, } 5 \\
\text { depths) }\end{array}$ & 20 & $10-30$ & $1 \mathrm{~N} \mathrm{NaOH}$ & \\
\hline $\begin{array}{l}\text { Soil of virgin tropical } \\
\text { forest }\end{array}$ & Mahé, Seychelles & $\begin{array}{l}3 \text { (1 location, } 3 \\
\text { depths) }\end{array}$ & 3 & $1-5$ & & \\
\hline Sewage sludge, raw & Germany & 3 plants & 97 & $65-135$ & & \\
\hline $\begin{array}{l}\text { Sewage sludge, } \\
\text { digested }\end{array}$ & Germany & 2 plants & 118 & $85-150$ & & \\
\hline Dewatered sludge & Germany & 3 plants & 85 & $10-220$ & & \\
\hline Manure, cattle & Germany, 1 plant & $\begin{array}{l}24 \text { ( } 4 \text { seasons, } 6 \\
\text { stages) }\end{array}$ & 802 & $9.53-9021$ & $1 \mathrm{~N} \mathrm{H}_{2} \mathrm{SO}_{4}$ & \\
\hline Manure, swine & Germany, 2 plants & $\begin{array}{l}36 \text { (4 seasons, } \\
11 \text { stages) }\end{array}$ & 1626 & $16-11550$ & & \\
\hline Stone minerals & Mineral collection & 11 minerals & 1818 & $11-6672$ & $1 \mathrm{~N} \mathrm{HCl}$ & \\
\hline $\begin{array}{l}\text { Soil of paddy field, } \\
\text { flooded }\end{array}$ & Beijing, China & $\begin{array}{l}4 \text { (1 location, } 4 \\
\text { depths) }\end{array}$ & 9 & $2-13$ & $1 \mathrm{~N} \mathrm{H}_{2} \mathrm{SO}_{4}$ & Liu et al. (1999) \\
\hline $\begin{array}{l}\text { Sediment of water } \\
\text { reservoir }\end{array}$ & Beijing, China & $\begin{array}{l}4 \text { (1 location, } 4 \\
\text { depths) }\end{array}$ & 3 & $2-4$ & & \\
\hline $\begin{array}{l}\text { Soil of paddy field, } \\
\text { drainage after } \\
\text { tilling }\end{array}$ & Beijing, China & $\begin{array}{l}11 \text { (1 location, } \\
11 \text { depths) }\end{array}$ & 2 & $0-7$ & $1 \mathrm{~N} \mathrm{H}_{2} \mathrm{SO}_{4}$ & Han et al. (2000) \\
\hline $\begin{array}{l}\text { Soil of paddy field, } \\
\text { drainage before } \\
\text { harvest }\end{array}$ & Beijing, China & $\begin{array}{l}10 \text { (1 location, } \\
10 \text { depths) }\end{array}$ & 3 & $0-11$ & & \\
\hline Manure, cattle & Germany & 1 & 14 & 14 & $\begin{array}{l}10 \% \\
\mathrm{NaOH}\end{array}$ & $\begin{array}{l}\text { Gassmann and } \\
\text { Glindemann (1993) }\end{array}$ \\
\hline Manure, swine & Germany & 1 & 964 & 964 & & \\
\hline $\begin{array}{l}\text { Sewage sludge, before } \\
\text { incubation }\end{array}$ & USA & 11 locations & 74 & $8-204$ & $10 \% \mathrm{NaOH}$ & Devai et al. (1999) \\
\hline $\begin{array}{c}\text { Sewage sludge, after } 7 \\
\text { days of incubation }\end{array}$ & USA & 11 locations & 545 & $111-2053$ & $10 \% \mathrm{NaOH}$ & Devai et al. (1999) \\
\hline
\end{tabular}

possible production. Therefore, the concentration of phosphine did not increase with time in the analysed soil samples. "Matrix-bound" phosphine, however, can always be found in soil and sludge samples. Therefore, matrix phosphine in biological samples may be a stationary state concentration of phosphine between production and consumption. Even if this phosphine turnover were important, only a small residue of 
Table 2

Free phosphine in gas products of the biosphere

\begin{tabular}{|c|c|c|c|c|}
\hline Sample & Location & Independent samples $(n)$ & Phosphine, range $\left(\mathrm{ng} \mathrm{m}^{-3}\right)$ & References \\
\hline $\begin{array}{l}\text { Biogas from } \\
\text { sediments of } \\
\text { sewage plants and } \\
\text { shallow lakes }\end{array}$ & Hungary & 11 & $11600000-382000000$ & Devai et al. (1988) \\
\hline Landfill gas & $\begin{array}{l}\text { Germany, } \\
\text { 1994-1995 }\end{array}$ & 36 (1 plant, 4 seasons) & $0-17731$ & Glindemann et al. (1996b) \\
\hline $\begin{array}{l}\text { Composting gas, } \\
\text { municipal waste }\end{array}$ & & 14 (2 plants, 2 seasons) & $22-341$ & \\
\hline $\begin{array}{l}\text { Composting gas, } \\
\text { cattle manure } \\
\text { biosolids }\end{array}$ & & 3 (seasons) & $0-55$ & \\
\hline $\begin{array}{l}\text { Composting gas, } \\
\text { swine manure } \\
\text { biosolids }\end{array}$ & & 4 (seasons) & $31-1015$ & \\
\hline $\begin{array}{l}\text { Interstitial gas, stored } \\
\text { sewage sludge, } \\
\text { limed }\end{array}$ & & 8 (2 plants) & $833-23233$ & \\
\hline $\begin{array}{l}\text { Interstitial gas, stored } \\
\text { sewage sludge, } \\
\text { unlimed }\end{array}$ & & 3 (1 plant) & $9-29$ & \\
\hline $\begin{array}{l}\text { Biogas of sewage } \\
\text { plant }\end{array}$ & & 16 (4 plants, 4 seasons) & $0-6167$ & \\
\hline $\begin{array}{l}\text { Marsh gas of Elster } \\
\text { river }\end{array}$ & & 6 (3 locations, 2 seasons) & $0-4$ & \\
\hline $\begin{array}{l}\text { Biogas of cattle } \\
\text { manure }\end{array}$ & & 4 (seasons) & $0-13$ & \\
\hline $\begin{array}{l}\text { Putrefaction gas of } \\
\text { cattle manure } \\
\text { storage }\end{array}$ & & 3 (seasons) & $123-238$ & \\
\hline $\begin{array}{l}\text { Biogas, swine manure } \\
\text { digestion }\end{array}$ & & 4 (seasons) & $0-1623$ & \\
\hline $\begin{array}{l}\text { Putrefaction gas, } \\
\text { swine manure } \\
\text { storage }\end{array}$ & & 14 (2 plants, 4 seasons) & $51-8995$ & \\
\hline $\begin{array}{l}\text { Biogas, putrefaction } \\
\text { gas, } 3 \text { swine and } \\
\text { cattle farming } \\
\text { plants }\end{array}$ & Germany, 1996 & 50 (3 plants, 4 seasons) & $2-47000$ & Glindemann (unpublished) \\
\hline Landfill gas & Beijing, China & 11 (wells) & $32-1062$ & Liu et al. (1999) \\
\hline $\begin{array}{l}\text { Marsh gas, paddy } \\
\text { field }\end{array}$ & Beijing, China & 4 & $26-41$ & \\
\hline $\begin{array}{l}\text { Marsh gas, water } \\
\text { reservoir }\end{array}$ & Beijing, China & 4 & $44-135$ & \\
\hline $\begin{array}{l}\text { Monoseptic bacterial } \\
\text { culture headspace }\end{array}$ & UK & 12 (bacterial strains) & $0-76000$ & Jenkins et al. (2000) \\
\hline $\begin{array}{l}\text { Faeces mixed bacterial } \\
\text { culture headspace }\end{array}$ & UK & 6 & $36000-720000$ & \\
\hline $\begin{array}{l}\text { Flatus gas of human } \\
\text { colon, } 2 \text { males, } \\
\text { meat rich diet }\end{array}$ & UK & 4 & $0-40000$ & Chughtai et al. (1998) \\
\hline $\begin{array}{l}\text { Headspace in sewage } \\
\text { sludge inoculated } \\
\text { media }\end{array}$ & Switzerland & 2 & $113-125$ & Rutishauser and Bachofen (1999) \\
\hline
\end{tabular}


Table 3

Emission flux of phosphine from the biosphere

\begin{tabular}{llllr}
\hline Origin & Location & $\begin{array}{l}\text { Emission } \\
\left(\mathrm{ng} \mathrm{m}^{-2} \mathrm{~h}^{-1}\right)\end{array}$ & References & $\begin{array}{r}\text { Number of } \\
\text { samples }(n)\end{array}$ \\
\hline Soil of brackish marsh & Louisiana, USA & $0.42-3$ & Devai and DeLaune (1995) & 2 \\
Louisiana salt marsh & & $0.91-6.52$ & Devai and DeLaune (1995) & 2 \\
Soil of paddy field & Beijing, China & -13 to +22 & Han et al. (2000) & Gassmann (2001, personal communication) \\
Intertidal mud flat area & North Sea, German bight & 4500 & G & \\
\hline
\end{tabular}

Table 4

Phosphine in the atmosphere at locations around the world (height $1 \mathrm{~m}$ above ground, sampled at night between 10 p.m. and 6 a.m., unless otherwise indicated)

\begin{tabular}{|c|c|c|c|c|c|}
\hline \multirow[t]{2}{*}{ Sampling location } & \multirow[t]{2}{*}{ Sampling time } & \multirow{2}{*}{$\begin{array}{l}\text { Number of } \\
\text { samples }(n)\end{array}$} & \multicolumn{2}{|c|}{ Phosphine $\left[\mathrm{pg} \mathrm{m}^{-3}\right]$} & \multirow[t]{2}{*}{ Source } \\
\hline & & & Average & Range & \\
\hline $\begin{array}{l}\text { Germany, urban location in } \\
\text { Berlin }\end{array}$ & 18 September 1995 & 2 locations & 157050 & $135380-178700$ & \\
\hline Germany, urban area in Leipzig & 21 September 1994 & 6 locations & 4630 & $3930-5900$ & \\
\hline $\begin{array}{l}\text { Germany, rural area east of } \\
\text { Leipzig }\end{array}$ & 21 September 1994 & 13 locations & 2030 & $1110-3420$ & \\
\hline Germany, urban area in Hamburg & 5 October 1994 & 5 locations & 2030 & $650-4490$ & \\
\hline $\begin{array}{l}\text { Germany, rural area west of } \\
\text { Stuttgart }\end{array}$ & 13 October 1994 & 6 locations & 1520 & $490-3060$ & \\
\hline $\begin{array}{l}\text { Germany, Leipzig, } 120 \mathrm{~m} \text {, top of } \\
\text { university tower }\end{array}$ & $\begin{array}{l}\text { September } \\
\text { 1994-July } 1995\end{array}$ & 8 days & 480 & $0-980$ & Glindemann et al. (1996) \\
\hline $\begin{array}{l}\text { Germany, urban location in } \\
\text { Leipzig }\end{array}$ & $\begin{array}{l}\text { September } \\
\text { 1994-July } 1995\end{array}$ & 77 days & 850 & $0-8880$ & \\
\hline $\begin{array}{l}\text { Germany, urban location in } \\
\text { Leipzig, } 12 \text { a.m. }\end{array}$ & $\begin{array}{l}\text { September } \\
\text { 1994-July } 1995\end{array}$ & 77 days & 116 & $0-1720$ & \\
\hline $\begin{array}{l}\text { Argentina, urban area in Buenos } \\
\text { Aires }\end{array}$ & 11 April 1995 & 5 locations & 620 & $320-1200$ & \\
\hline $\begin{array}{l}\text { Tunisia, rural location near } \\
\text { Hammamet }\end{array}$ & 23 March 1995 & 6 locations & 510 & $0-1150$ & \\
\hline Seychelles, Mahé & April 1995 & 13 locations & 280 & $0-410$ & \\
\hline $\begin{array}{l}\text { Israel, rural area, Ein Bokek and } \\
\text { Rishon Lezion }\end{array}$ & 19, 22 January 1995 & 4 locations & 130 & $0-300$ & \\
\hline Namibia, rural area & March 1995 & 5 locations & 40 & $0-280$ & \\
\hline $\begin{array}{l}\text { High altitude } 12,500 \mathrm{~m} \text {, trans } \\
\text { North Atlantic flight with } \\
\text { research airplane }\end{array}$ & $\begin{array}{l}24-25 \text { November } \\
1995\end{array}$ & $\begin{array}{l}12 \text { loca- } \\
\text { tions/heights }\end{array}$ & 956 & $200-2450$ & Glindemann (unpublished) \\
\hline Germany, marine air, North Sea & Summer 1996 & 9 locations & 250 & 41-855 & Gassmann (1994) \\
\hline $\begin{array}{l}\text { China, Beijing, urban residential } \\
\text { area }\end{array}$ & Summer 1997 & 10 days & 14890 & $2600-64800$ & Liu et al. (1999) \\
\hline $\begin{array}{l}\text { China, Beijing, urban residential } \\
\text { area }\end{array}$ & Winter 1997 & 10 days & 1230 & $0-2700$ & \\
\hline $\begin{array}{l}\text { China, Beijing, urban residential } \\
\text { area, } 12 \text { a.m. }\end{array}$ & Summer 1997 & 10 days & 1100 & $0-11000$ & \\
\hline $\begin{array}{l}\text { China, Beijing, urban residential } \\
\text { area, } 12 \text { a.m. }\end{array}$ & Winter 1997 & 10 days & 970 & $0-4000$ & \\
\hline $\begin{array}{l}\text { Beijing, tower, high altitude, } 0 \text { to } \\
300 \mathrm{~m} \text { above ground }\end{array}$ & 25 March 1998 & 7 heights & 1414 & $1100-2300$ & \\
\hline China, near Beijing, paddy field & 25 June 1997 & 3 locations & 137290 & $127220-145860$ & \\
\hline
\end{tabular}
(adjacent air), 12 a.m. 
Table 4 (Continued)

\begin{tabular}{|c|c|c|c|c|c|}
\hline \multirow[t]{2}{*}{ Sampling location } & \multirow[t]{2}{*}{ Sampling time } & \multirow{2}{*}{$\begin{array}{l}\text { Number of } \\
\text { samples }(n)\end{array}$} & \multicolumn{2}{|c|}{ Phosphine $\left[\mathrm{pg} \mathrm{m}^{-3}\right.$ ] } & \multirow[t]{2}{*}{ Source } \\
\hline & & & Average & Range & \\
\hline $\begin{array}{l}\text { China, near Beijing, water } \\
\text { reservoir (adjacent air), } 12 \text { a.m. }\end{array}$ & 25 June 1997 & 4 locations & 97970 & 50120-166280 & \\
\hline $\begin{array}{l}\text { China, Beijing, urban landfill site } \\
\text { (adjacent air), } 12 \text { a.m. }\end{array}$ & 27 June 1997 & 11 locations & 14020 & $1080-71000$ & \\
\hline $\begin{array}{l}\text { China, Beijing, paddy field } \\
\text { (adjacent air) }\end{array}$ & $\begin{array}{l}25 \text { June } 1999-28 \\
\text { September } 1999\end{array}$ & 4 days & 42000 & $2500-100000$ & Han et al. (2000) \\
\hline $\begin{array}{l}\text { China, Beijing, paddy field } \\
\text { (adjacent air), } 12 \text { a.m. }\end{array}$ & $\begin{array}{l}25 \text { June } 1999-28 \\
\text { September } 1999\end{array}$ & 4 days & 4500 & $1000-7000$ & \\
\hline
\end{tabular}

this turnover could be observed as matrix-bound phosphine.

Under such circumstances, a slow migration process of phosphine in the interstitial gas sphere of soils is possible. Such a process would influence the balance of phosphorus in agricultural and wetland soils. To our knowledge, no experiments have ever been undertaken to document phosphine migration in soil.

In biogases, the concentrations of phosphine is in the range $\mathrm{ng} \mathrm{m}^{-3}$ to $\mu \mathrm{g} \mathrm{m}^{-3}$ (Table 2). No other form of volatile phosphorus has been reported or detected in biogases. Devai et al. (1988) found exceptionally high values, but the number of measurements performed was relatively small.

The number of measured fluxes of phosphine (Table 3) is almost insufficient to draw any conclusions. The biogas concentrations in Table 1 can be taken to estimate flux because the quantity of biogas flux can be estimated for sites such as swamps and biogas digesters.

In atmospheric air, the concentration of phosphine is in the range $\mathrm{pg} \mathrm{m}^{-3}$ to $\mathrm{ng} \mathrm{m}^{-3}$ (Table 4). The reported concentration of phosphine in China is significantly higher near paddy fields than in urban residential areas. Phosphine has been measured in the atmosphere at altitudes of 120,300 and $12,500 \mathrm{~m}$.

The data of phosphine in atmospheric air in Table 4 indicate important sources and a flux of phosphine, which accumulates in the night atmosphere and decreases until noon of the following day through oxidation (oxidation mechanism by solar radiation and OH radicals see Frank and Rippen, 1987; WHO, 1988). This could explain the finding of soluble phosphate in rain water (Lewis et al., 1985). Over the long term this flux could be important for the long-range transport of phosphorus to remote places.
Compared to the large number of studies on the occurrence of phosphates, the historical data presented in this review on phosphine are still insufficient to draw final conclusions. Nevertheless, the numbers presented can be used to draw a preliminary picture. The reviewed data support the hypothesis of the existence of a small gaseous link to the phosphorus cycle. This gaseous phosphorus cycling including the migration of phosphine within different soil compartments and the exchange of phosphorus between soil and the atmosphere could become important over the long term.

\section{Acknowledgement}

Supported by the NSF (grant no. BES-0201849). The opinions expressed are those of the authors and not necessarily those of the Foundation.

\section{References}

Bolin, B., Cook, R.B., 1983. The Major Biogeochemical Cycles and Their Interactions. John Wiley \& Sons, New York, NY, p. 327.

Cao, H.F., Liu, J., Zhuang, Y.H., Glindemann, D., 2000. Emission sources of atmospheric phosphine and simulation of phosphine formation. Sci. China Ser. B. 43, 162-168.

Chughtai, M., Pridham, P.B., Gates, P.N., Cooke, M., 1998. Determination of Phosphine by packed column gas chromatography with alkali flame ionisation detection. Anal. Commun. 35, 109-111.

Devai, I., DeLaune, R.D., 1995. Evidence for phosphine production and emission from Louisiana and Florida marsh soils. Org. Geochem. 23, 277-279.

Devai, I., DeLaune, R.D., Devai, G., Patrick, W.H., Czegeny, I., 1999. Phosphine production potential of various wastewater and sewage sludge sources. Anal. Lett. 32, 1447-1457.

Devai, I., Felföldy, L., Wittner, I., Plosz, S., 1988. Detection of phosphine: new aspects of the phosphorus cycle in the hydrosphere. Nature 333, 343-345. 
Devai, I., Wittner, I., Felföldy, L., Plosz, S., 1984. Process for freeing from phosphate the solutions containing phosphate, via microbiological way, and purifying the phosphates. Hungarian Patent 194129.

Eismann, F., Glindemann, D., Bergmann, A., Kuschk, P., 1997. Soils as a source and sink of phosphine. Chemosphere 35, 523-533.

Emsley, J., 2000. The 13th Element: The Sordid Tale of Murder, Fire, and Phosphorus. John Wiley \& Sons, New York, NY, p. 327.

Frank, R., Rippen, G., 1987. Verhalten von Phosphin in der Atmosphaere. Lebensmitteltechnik 17, 409-411.

Gassmann, G., 1994. Phosphine in the fluvial and marine hydrosphere. Mar. Chem. 45, 197-205.

Gassmann, G., Glindemann, D., 1993. Phosphane in the Biosphere. Angew. Chem. Int. Ed. 32, 761-763.

Gassmann, G., Glindemann, D., van Beusekom, J., 1996. Offshore atmospheric phosphine. Naturwissenschaften 83, 129-131.

Gassmann, G., Schorn, F., 1993. Phosphine from harbour surface sediments. Naturwissenschaften $80,78-80$.

Glindemann, D., Bergmann, A., 1995. Spontaneous emission of phosphane from animal slurry treatment processing. Zentralblatt für Hygiene und Umweltmedizin 198, 49-56.

Glindemann, D., Bergmann, A., Stottmeister, U., Gassmann, G., 1996a. Phosphine in the lower terrestrial troposphere. Naturwissenschaften 83, 131-133.

Glindemann, D., Stottmeister, U., Bergmann, A., 1996b. Free phosphine from the anaerobic biosphere. Environ. Sci. Pollut. Res. 3, 17-19.

Glindemann, D., Eismann, F., Bergmann, A., Kuschk, P., Stottmeister, U., 1998. Phosphine by bio-corrosion of phosphide-rich iron. Environ. Sci. Pollut. Res. 5, 71-74.
Graham, W.F., Duce, R.A., 1979. Atmospheric pathways of the phosphorus cycle. Geochim. Cosmochim. A 43, 1195-1208.

Han, S.H., Zhuang, Y.H., Liu, J.A., Glindemann, D., 2000. Phosphorus cycling through phosphine in paddy fields. Sci. Total Environ. 258, 195-203.

Iverson, W.P., 1968. Corrosion of iron and formation of iron phosphide by Desulfovibrio desulfuricans. Nature 217, 12651267.

Jenkins, R.O., Morris, T.A., Craig, P.J., 2000. Phosphine generation by mixed- and monoseptic-cultures of anaerobic bacteria. Sci. Total Envrion. 250, 73-81.

Lewis, W.M., Grant, M.C., Hamilton, S.K., 1985. Evidence, that filterable phosphorus is a significant atmospheric link in the phosphorus cycle. Oikos 45, 428-432.

Liu, J.A., Zhuang, Y.H., Kuschk, P., Eismann, F., Glindemann, D., 1999. Phosphine in the urban air of Beijing and its possible sources. Water Air Soil Pollut. 116, 597-604.

Nowicki, T.W., 1978. Gas-Liquid chromatography and flame photometric detection of phosphine in wheat. J. Assoc. Off. Anal. Chem. 61, 829-836.

Prinn, R.G., Lewis, J.S., 1975. Phosphine on Jupiter and implications for the great red spot. Science 190, 274-276.

Roels, J., Verstraete, W., 2001. Biological formation of volatile phosphorus compounds: a review. Biorsour. Technol. 79, 243-250.

Rutishauser, B.V., Bachofen, R., 1999. Phosphine formation from sewage sludge cultures. Anaerobe 5, 525-531.

Tsubota, G., 1959. Phosphate reduction in the paddy field. Soil Plant Food Tokyo 5, 10-15.

World Health Organization, 1988. Phosphine and selected metal phosphides. In: Environmental Health Criteria, vol. 73, Geneva. 